\title{
Corrigendum
}

\section{Corrigendum to "Physiological and molecular mechanisms associated with cross tolerance between hypoxia and low temperature in Thaumatotibia leucotreta" [J. Insect Physiol. 82 (2015) 75-84]}

\author{
Leigh Boardman ${ }^{\mathrm{a}, *}$, Jesper G. Sørensen ${ }^{\mathrm{b}}$, John S. Terblanche ${ }^{\mathrm{a}}$ \\ ${ }^{a}$ Department of Conservation Ecology and Entomology, Centre for Invasion Biology, Stellenbosch University, Private Bag X1, Matieland 7602, South Africa \\ ${ }^{\mathrm{b}}$ Section for Genetics, Ecology \& Evolution, Department of Bioscience, Aarhus University, Ny Munkegade 116, DK-8000 Aarhus C, Denmark
}

The authors regret that there was an error in the illustration of the results of the total protein assay, resulting in incorrect representations of this variable in Figs. 2h, 3, Table 2 and Supplementary data in the published version of the paper. These errors have now been corrected and the corrections to the relevant discussion points are noted below. The overall conclusions and outcomes of the study are however unchanged despite this error.

Table 2: Mechanical stress, acute hypoxia, chronic dry hypoxia and chronic moist hypoxia pre-treatments increased total protein relative to the handling control at the timepoints indicated.

3.3. Cross tolerance hypothesis: The start of the second paragraph should read: "More specifically, the non-thermal, non-gas mechanical stress pre-treatment was associated with an increase in total protein concentration, HSP70 and sorbitol (Table 1; Fig. S1h, i, m)."

3.6. Principal components analysis: Factor 1 explains $41.83 \%$ of the variance, and factor 2 explains $23.32 \%$ (see Fig. 3 ). According to the revised PCA, total protein did not change in response to low temperatures.

The authors would like to apologize for any inconvenience caused.

\section{Appendix A. Supplementary data}

Supplementary data associated with this article can be found, in the online version, at http://dx.doi.org/10.1016/j.jinsphys.2016.05.004.

DOI of original article: http://dx.doi.org/10.1016/j.jinsphys.2015.09.001

* Corresponding author.

E-mail address: boardman.leigh@gmail.com (L. Boardman). 

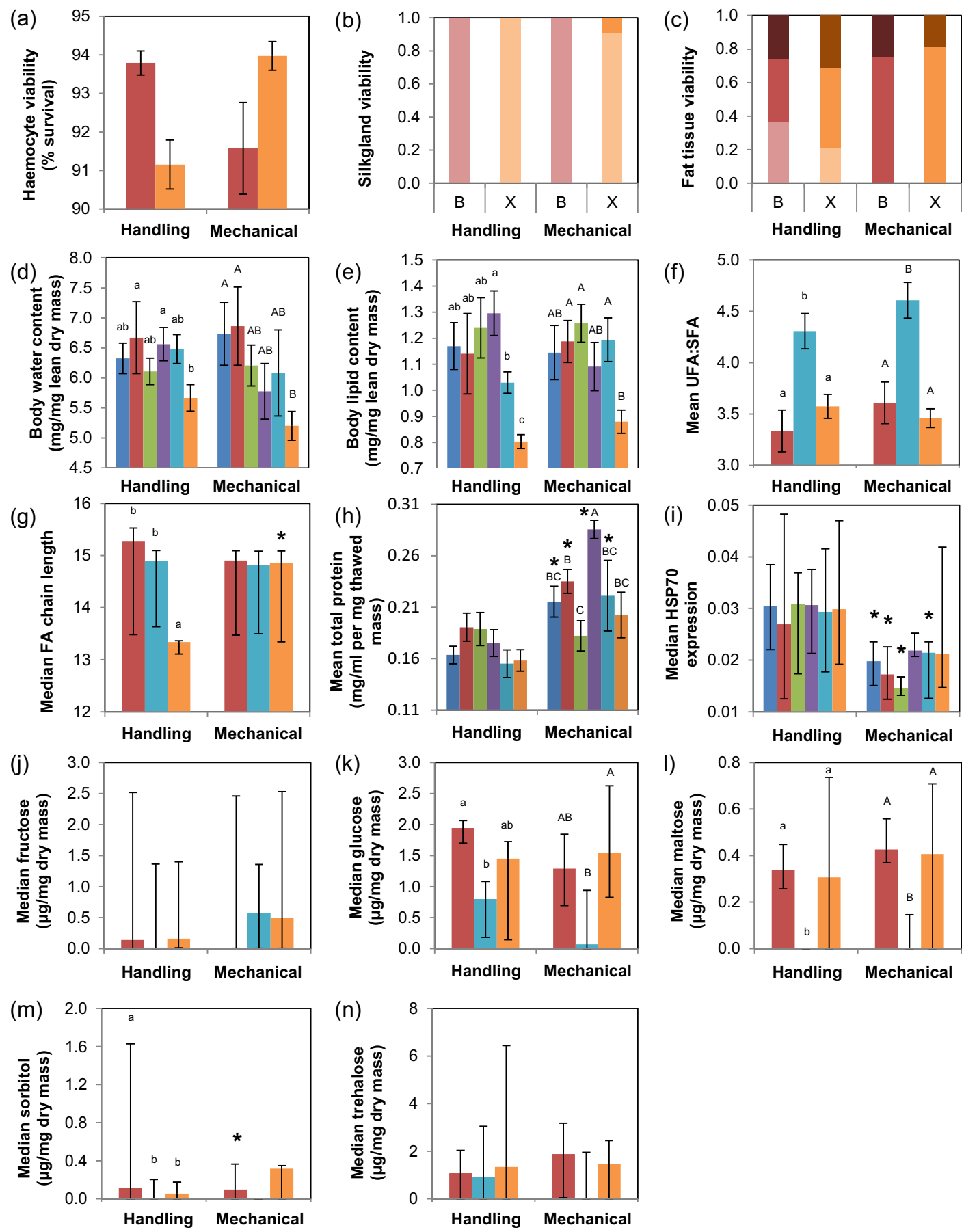

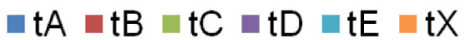

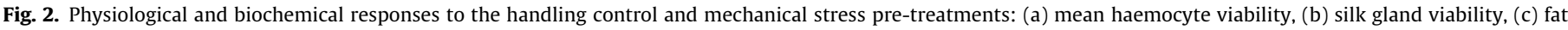

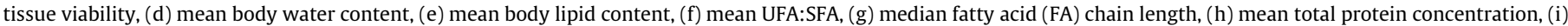

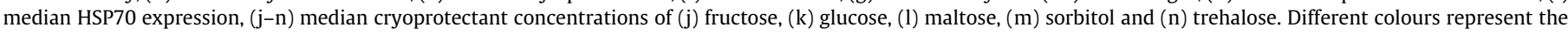

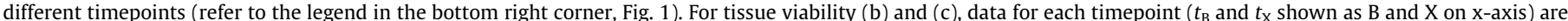

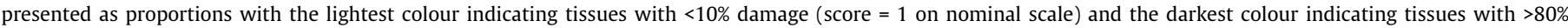

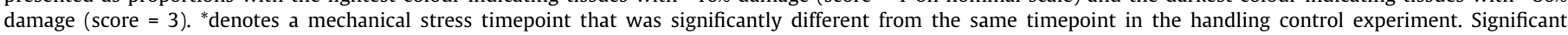

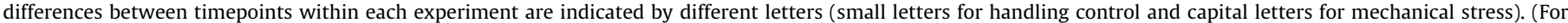
interpretation of the references to colour in this figure legend, the reader is referred to the web version of this article.) 
Table 2

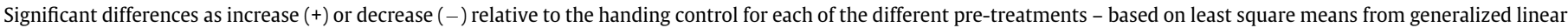

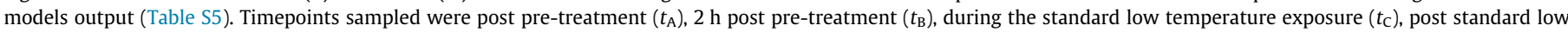
temperature exposure $\left(t_{\mathrm{D}}\right), 2 \mathrm{~h}$ post standard low temperature exposure $\left(t_{\mathrm{E}}\right)$ and $26 \mathrm{~h}$ post standard low temperature exposure $\left(t_{\mathrm{X}}\right)$.

\begin{tabular}{|c|c|c|c|c|c|c|c|}
\hline Variable $^{a}$ & Timepoint & Mechanical stress & Low temperature & High temperature & Acute hypoxia & Chronic dry hypoxia & Chronic moist hypoxia \\
\hline Survival & $t_{\mathrm{X}}$ & & + & & + & & + \\
\hline \multirow[t]{2}{*}{ Haemocyte viability } & $t_{\mathrm{B}}$ & & & & & & \\
\hline & $t_{\mathrm{X}}$ & & & & & & - \\
\hline \multirow[t]{6}{*}{ Body water content } & $t_{\mathrm{A}}$ & & + & & - & & \\
\hline & $t_{\mathrm{B}}$ & & + & - & & - & - \\
\hline & $t_{\mathrm{C}}$ & & + & & - & - & \\
\hline & $t_{\mathrm{D}}$ & & + & & - & - & \\
\hline & $t_{\mathrm{E}}$ & & + & & - & & - \\
\hline & $t_{\mathrm{X}}$ & & + & & - & & \\
\hline \multirow[t]{6}{*}{ Body lipid content } & $t_{\mathrm{A}}$ & & & & & & \\
\hline & $t_{\mathrm{B}}$ & & & & & & \\
\hline & $t_{\mathrm{C}}$ & & + & & & & \\
\hline & $t_{\mathrm{D}}$ & & & & & & \\
\hline & $t_{\mathrm{E}}$ & & & + & & & \\
\hline & $t_{\mathrm{X}}$ & & & & + & & + \\
\hline \multirow[t]{3}{*}{ UFA:SFA } & $t_{\mathrm{B}}$ & & + & & & + & \\
\hline & $t_{\mathrm{E}}$ & & & & & & \\
\hline & $t_{\mathrm{X}}$ & & & & & & \\
\hline \multirow[t]{3}{*}{ Fatty acid chain length } & $t_{\mathrm{B}}$ & & - & & & & \\
\hline & $t_{\mathrm{E}}$ & & & & & & \\
\hline & $t_{\mathrm{X}}$ & + & + & + & + & + & + \\
\hline \multirow[t]{6}{*}{ Total protein content } & $t_{\mathrm{A}}$ & + & & & + & & + \\
\hline & $t_{\mathrm{B}}$ & + & & & & & + \\
\hline & $t_{\mathrm{C}}$ & & & & + & & \\
\hline & $t_{\mathrm{D}}$ & + & & & + & + & + \\
\hline & $t_{\mathrm{E}}$ & + & & & + & & \\
\hline & $t_{\mathrm{X}}$ & & & & + & + & + \\
\hline \multirow[t]{6}{*}{ HSP70 } & $t_{\mathrm{A}}$ & - & & & - & & \\
\hline & $t_{\mathrm{B}}$ & - & + & & & & \\
\hline & $t_{\mathrm{C}}$ & - & & & & & \\
\hline & $t_{\mathrm{D}}$ & & & & - & & \\
\hline & $t_{\mathrm{E}}$ & - & + & & - & - & \\
\hline & $t_{\mathrm{X}}$ & & + & - & - & & \\
\hline \multirow[t]{3}{*}{ Sorbitol } & $t_{\mathrm{B}}$ & - & - & - & - & & - \\
\hline & $t_{\mathrm{E}}$ & & & & & & \\
\hline & $t_{\mathrm{X}}$ & & & & & & \\
\hline
\end{tabular}

${ }^{a}$ Results for pupation, emergence, silk gland viability, fat tissue viability, fructose, glucose, maltose or trehalose were not significantly different to the handling control at any timepoint.
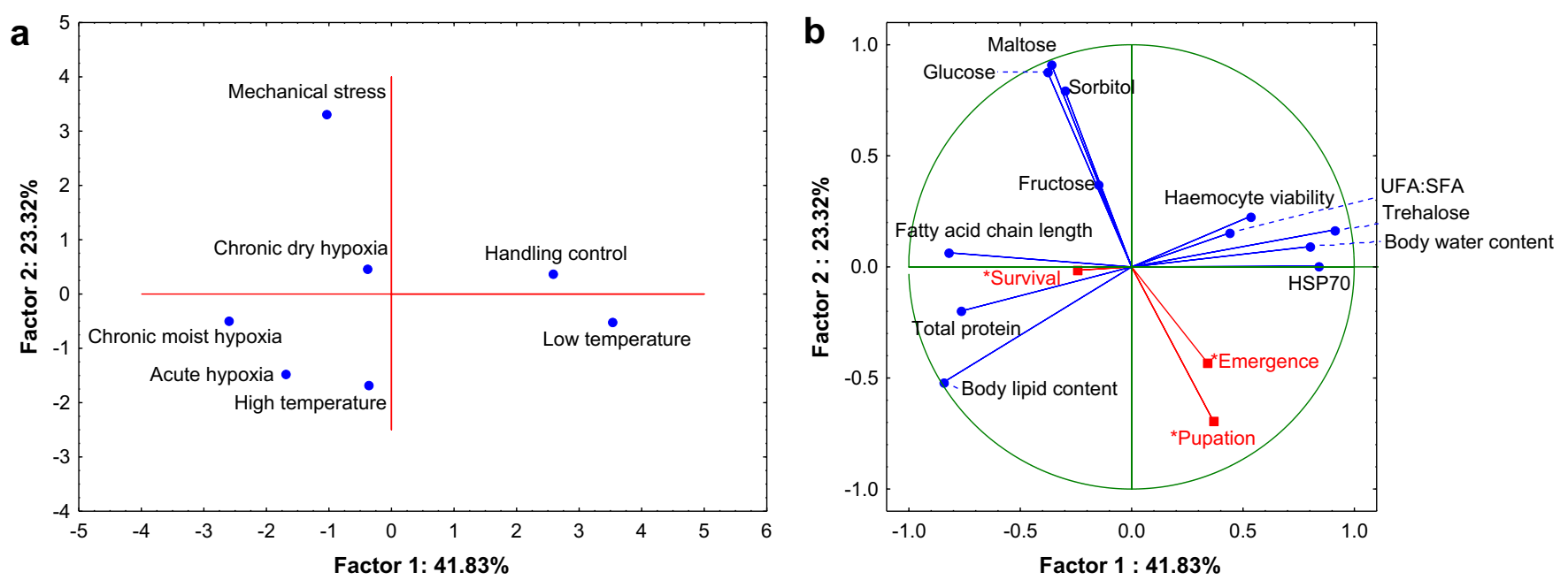

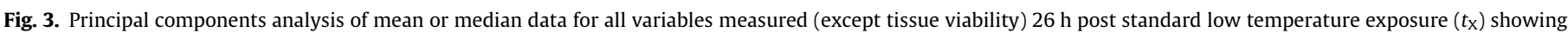

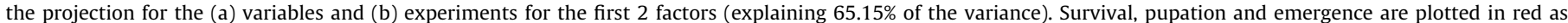

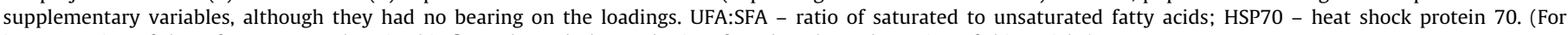
interpretation of the references to colour in this figure legend, the reader is referred to the web version of this article.) 